\title{
Terræ Didatica e Terræ agora são uma só, no ambiente Open Journal Systems
}

Terre Didatica and Terre are now one in the Open Journal Systems environment

Terre Didatica yTerre se unen como un solo medio de comunicación bajo el Open Journal Systems

Correspondência

Os editores

Terræ Didatica

Instituto de Geociências

Caixa Postal (P.O. Box) 6152

Rua Carlos Gomes 250,

13083-855 Campinas, SP, Brasil

E-mail: terraedidatica@ige.unicamp.br 
O estudo da Terra sob perspectiva sistêmica concilia o caráter complexo, experimental e simultaneamente histórico de Ciência do Sistema Terra, em um tratamento multidisciplinar que contribui na formação da cidadania. Fortalecendo o enorme potencial dos estudos da Terra para tratar de problemas que extrapolam os limites das disciplinas tradicionais, o desenvolvimento das especializações e áreas profissionais das Geociências sob o prisma dos sistemas terrestres representa importante objetivo de formação para estudantes em cursos que contemplem algum componente ambiental. Sobretudo nos campos de atuação profissional da Hidrogeologia, Geologia Urbana e Geologia de Recursos Energéticos, emergem questões complexas que exigem a capacidade de teorizar, resolver problemas e tratar de questões globais, envolvendo fatores naturais, experimentais e sociais. Em todos os níveis educacionais as diferentes dimensões do currículo devem contribuir para o exercício de tarefas e preparação dos futuros cidadãos em campos que envolvam as ciências naturais, experimentais e analíticas, bem como os campos humanos e sociais. Aqueles que se dedicam ao Ensino de Ciências da Terra necessitam refletir sobre modos de explorar questões transdisciplinares das Geociências para aprofundar as relações entre Engenharia, Matemática e Ciências Sociais (Shipley et al. 2017):

Ao considerar problemas de todos os campos de conhecimento, pode-se caracterizar questões de pesquisa que transcendem as disciplinas. Identificar estas questões que não sejam perceptíveis dentro de uma única disciplina e usar ideias importantes de outras disciplinas constituem uma contribuição que pode ser dada pelas Geociências (Shipley et al. 2017).

Tais concepções inspiraram os editores de Terræ e Terræ Didatica a reunir os dois títulos em um só a partir de 2018; isso ajuda a atender à demanda por investigações acadêmicas que: (a) aprofundem o conhecimento da natureza; (b) contribuam para reflexão legal e ambiental em torno de problemas de conservação da natureza; (c) abordem o ensino e a aprendizagem vinculados a formações profissionais específicas que interfiram nas áreas geológicas e de engenharia e, finalmente, (d) ajudem a formar tomadores de decisão no campo ambiental. Em outros termos, a pesquisa educacional iluminará outros campos de conhecimento e será beneficiada por essa afinidade. Publicadas regularmente desde o início em 2004 e 2005, as revistas enfrentam limitações econômicas e financeiras, o que contribui para concentrar esforços em torno de um só título do IG-Unicamp, em lugar de dois.

O novo perfil de Terræ Didatica, com periodicidade trimestral, possibilita publicar inovações e recursos de alta qualidade para difundir o conhecimento, tanto científico, quanto educacional. A expansão vigorosa dos últimos anos aumentou a capacidade de se divulgar contribuições originais, demandando fluxo editorial mais ágil em meio eletrônico. Como o fluxo de recepção de manuscritos é contínuo, passamos a utilizar o ambiente OJS (Open Journal Systems). Os autores devem consultar as normas de submissão; recomenda-se que se inscrevam no sistema $\mathrm{ORCiD}^{1}$, para agilizar o processo. Visitantes do endereço www.ige.unicamp. br/terraedidatica serão redirecionados para o link:

\section{https:/tperiodieos.sbu.tnieamp.br/ojs/index.php/tt/login,}

Edições em papel de Terræ Didatica são gratuitamente distribuídas a bibliotecas, instituições públicas e organizações não governamentais de geologia, geografia, ensino e estudos ambientais. Atingem, dentro de certos limites, acadêmicos, professores, profissionais e estudantes. A impressão gráfica foi suspensa em 2017, por prazo indeterminado. Por que não eliminar totalmente o papel? Além de abandonar o registro ISSN de revista científica, perderíamos publicidade em potencial, devido à norma que impede que revistas on-line publiquem anúncios de empresas e entidades federais, como Petrobras, Banco do Brasil, Finep e outras.

\section{Planejamento das edições de 2018 e os eventos de julho na Unicamp}

Os números de março e junho acolhem artigos de submissão espontânea. A convite do Programa de Pós-Graduação em Ensino e História de Ciências da Terra (PEHCT) do IG-Unicamp, a revista incluirá no volume de 2018 uma seleção dos artigos da $8^{\text {th }}$ Quadrennial Conference of the International Geoscience Education Organisation (IGEO) / VIII GeoSciEd 2018 e $8^{\circ}$ Simpósio Nacional sobre Ensino e História de Ciências da Terra / EnsinoGEO-2018. Os eventos buscam disseminar conhecimento de Geociências de qualidade, focalizando em especial a comunidade

1 ORCiD (Open Researcher and Contributor ID) é um identificador digital único, gratuito e persistente, que distingue cada acadêmico/pesquisador e elimina eventuais ambiguidades e semelhanças de nomes de autores. 
Expediente Terræ Didatica v. 14, n. 1, 2018

\section{Reitor:}

Marcelo Knobel

Coordenadoria Geral da Universidade:

Teresa Dib Zambon Atvars (Vice-Reitora)

Chefe de Gabinete:

Joaquim Murray Bustorff Silva

Pró-Reitores

Pós-Graduação: André Tosi Furtado

Pesquisa: Munir Salomão Skaf

Graduação: Eliana Martorano Amaral

Extensão e Assuntos Comunitários: Fernando

Augusto de Almeida Hashimoto

Desenvolvimento Universitário: Marisa Masumi

Beppu

Instituto de Geociências

Diretor: Sérgio Luiz Monteiro Salles-Filho

Diretor-associado: Márcio Antonio Cataia

Coordenação de Programas:

Pós-Graduação: Emilson Pereira Leite

Graduação: Wanilson Luiz Silva

Extensão: Roberto Greco

Terræ Didatica, 2018 - v.14 - n.1

Instituto de Geociências da Universidade Estadual de Campinas

Caixa Postal 6152 - CEP 13083-970 - Campinas, SP Brasil

Telefones: +55 1935214564 e 35214562 - Fax +55 1935214552

e-mail: terraedidatica@ige.unicamp.br

http://periodicos.sbu.unicamp.br/ojs/index.php/td www.ige.unicamp.br/terraedidatica

Em 2018, os editores das duas publicações periódicas do Instituto de Geociências da Unicamp dedicadas à difusão de pesquisas científicas (Terræ), materiais didáticos e recursos educativos (Terræ Didatica) decidiram realizar uma fusão. Além de melhorar o alcance editorial das revistas, a medida atende à demanda por investigações acadêmicas que: (a) aprofundem o conhecimento da natureza; (b) estimulem uma reflexão legal e ambiental quanto à conservação da natureza; (c) abordem o ensino e a aprendizagem vinculados a formações profissionais específicas que interfiram nas áreas geológicas e de engenharia e, finalmente, (d) ajudem a formar tomadores de decisão no campo ambiental. A pesquisa educacional valorizará outros campos de conhecimento, sendo beneficiada por essa afinidade. Cada edição de Terræ Didatica está disponível em formato eletrônico, buscando difundir e debater resultados de pesquisas nas áreas de Ciências da Terra, Geografia e Ciências do Ambiente, no Brasil e outros países (em particular a comunidade íberolatino-americana). E distribuída em formato impresso, periodicamente, de forma dirigida, a bibliotecas universitárias, instituições de governo e organizações não-governamentais das áreas de geologia, geografia e estudos ambientais. É também distribuída a universidades, escolas, profissionais e estudantes que assim o solicitarem. A revista publica ainda resultados parciais de projetos, revisões de livros, avaliações de materiais didáticos, relatórios de conferências, cursos e roteiros de campo.

Estrato Qualis/CAPES (2017)

Estrato Área de Avaliação

A2 ENSINO

B3 GEOGRAFIA

B4 CIÊNCIAS AMBIENTAIS

B4 GEOCIÊNCIAS

B4 INTERDISCIPLINAR

Indexadores:

-EZB - Electronic Journals Library

- Scholar Google

- Latindex

- Portal de Periódicos Eletrônicos Científicos

(Unicamp)

-Portal de Periódicos Eletrônicos em Geociências

(PPEGeo)

-PKP - Public Knowledge Project

Divulgação em Catálogos, Bibliotecas Virtuais e

Índices de métricas

- Catálogo Acervo (UNICAMP)

- Catálogo Coletivo Nacional (IBICT)

- Scholar Google - Metrics (Índice H)

- WorldCat (OCLC)

\section{Editor-chefe}

Celso Dal Ré Carneiro - Inst. Geociências/Unicamp Editor-associado

Pedro Wagner Gonçalves - Inst. Geociências/Unicamp

Conselho Consultivo

Adalberto Scortegagna - Centro Universitário

Franciscano do Paraná, Curitiba, PR

Adejardo Francisco Silva Filho - Departamento de Geologia, Universidade Federal de Pernambuco,

UFPE, Recife, PE

Alfonso García DelaVega - Universidad Autônoma de Madrid, Espanha

Andre Sampaio Mexias - Instituto de Geociências,

Universidade Federal do Rio Grande do Sul, UFRGS, Porto Alegre, RS

Angela Beatriz Menezes Leal - Instituto de

Geociências, Universidade Federal da Bahia, UFBA,

Salvador, BA

Antonio José Teixeira Guerra - Instituto de Geociências, Universidade Federal do Rio de Janeiro, UFRJ, Rio de Janeiro, RJ

Benjamin Bley de Brito Neves - Instituto de

Geociências, Universidade de São Paulo, USP, São

Paulo, SP

Carlos Eduardo Barros - Departamento de

Geociências, Universidade Federal do Paraná, UFPR, Curitiba PR

Carlos Schobbenhaus Filho - CPRM Serviço

Geológico do Brasil, Brasília DF

Clara Maria da Silva de Vasconcelos - Universidade do Porto, Porto Portugal

Chan-Jong Kim - Seoul National University, South

Korea

Chris King - Keele University, Staffordshire, UK

David Brusi - Universidade de Girona, Girona, Espanha

\begin{tabular}{c|c|c|c|c|c|c|}
\hline (C) Terrae Didat. & Campinas, SP & v.14 & n.1 & p. 3-4 & jan./mar. 2018 \\
\hline \multicolumn{4}{|c|}{3}
\end{tabular}


Didier Gastmans - Instituto de Geociências e Ciências Exatas, Universidade Estadual Paulista, UNESP, Rio Claro, SP

Dimas Dias Brito - Instituto de Geociências e Ciências Exatas, Universidade Estadual Paulista, UNESP, Rio Claro, SP

Eduardo Soares de Macedo - Instituto de Pesquisas Tecnológicas, IPT, São Paulo, SP

Elvo Fassbinder - Departamento de Geociências, Universidade Federal do Paraná, UFPR, Curitiba, PR Francisco de Assis Matos de Abreu - Centro Geociências, UFPA, Universidade Federal do Pará, Belém, PA

Geraldo Norberto Chaves Sgarbi - Instituto de Geociências, UFMG, Universidade Federal de Minas Gerais, Belo Horizonte, MG

Gillian Drennan - Witwatersrand University, Johannesburg, South Africa

Giorgio Basilici - Instituto de Geociências,

Universidade Estadual de Campinas, Unicamp, Campinas, SP

Greg McNamara - Geological Society of Australia, Camberra, Australia

Hector Luis Lacreu - Universidad de San Luis, San Luis, Argentina

Ismar de S Carvalho - Instituto de Geociências, Universidade Federal do Rio de Janeiro, UFRJ, Rio de Janeiro, RJ

Jorge Bonito - Universidade de Évora, Évora, Portugal Jorge Megid - Faculdade de Educação, Universidade Estadual de Campinas, Unicamp, Campinas, SP José Brilha - Universidade do Minho, Portugal Jose Eduardo Zaine - Instituto de Geociências e Ciências Exatas, Universidade Estadual Paulista, UNESP, Rio Claro, SP

Jose Fernando Pina Assis - Centro de Geociências, UFPA, Universidade Federal do Pará Belém PA José Sellés Martínez - Universidade de Buenos Aires, Buenos Aires, Argentina

Juan Gabriel Morcillo - Universidad Complutense de Madrid, Espanha

Katia L Mansur - Instituto de Geociências, UFRJ, Rio de Janeiro, RJ

Lázaro Valentim Zuquette - Escola de Engenharia de

São Carlos USP, São Carlos, SP

Leonor Bonan - Universidade de Buenos Aires, Buenos Aires, Argentina

Luís Marques - Universidade de Aveiro, Aveiro, Portugal

María Amelia Calonge García - Universidade de

Alicante, Espanha

Maria Cristina Motta de Toledo - Escola de Artes,

Ciências e Humanidades, Universidade de São Paulo, USP, São Paulo, SP

Marjorie Cseko Nolasco - Universidade Estadual de Feira de Santana, UEFS, Feira de Santana, BA
Michelle Simões Reboita - Universidade Federal de Itajubá, Itajubá, $\mathrm{MG}$

Miguel A Tupinamba A Souza - Faculdade de Geologia, Universidade do Estado do Rio de Janeiro, UERJ, Rio de Janeiro, RJ

Natalina Sicca - Centro Universitário Moura Lacerda,

Ribeirão Preto, SP

Oscar Braz Mendonza Negrao - Instituto de

Geociências, Universidade Estadual de Campinas,

Unicamp, Campinas, SP

Roberto Greco - Instituto de Geociências,

Universidade Estadual de Campinas, Unicamp,

Campinas, SP

Roberto Nardi - Departamento de Educação,

Faculdade de Ciências, Universidade Estadual Paulista, UNESP, Bauru, SP

Rualdo Menegat - Instituto de Geociências, UFRGS, Porto Alegre, RS

Michael J. Passow - Lamont-Doherty Earth

Observatory, Englewood NJ, EUA

Valderez Pinto Ferreira - Departamento de Geologia,

Universidade Federal de Pernambuco, UFPE, Recife, PE

Zorano Sergio de Souza - Departamento de Geologia, Universidade Federal do Rio Grande do Norte,

UFRN, Natal, RN

\section{Apoio editorial, produção gráfica e capa \\ Editora Beca}

\section{Colaboração na revisão em Inglês}

Cleveland M. Jones, Inst. Nac. de Óleo e Gás/CNPq, INORJ/UERJ

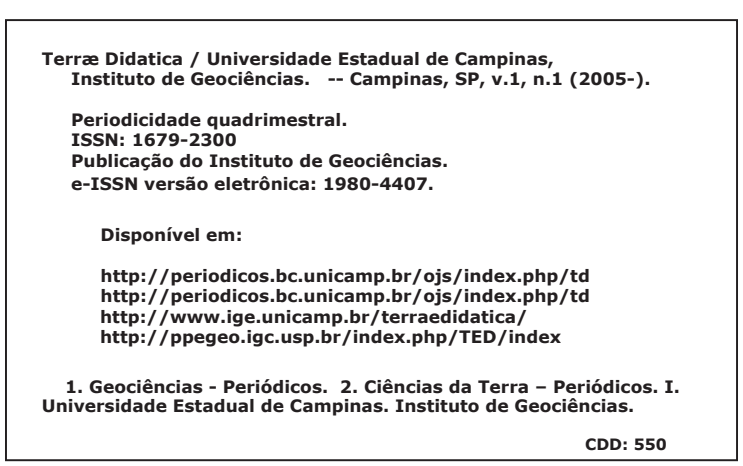

Imagem da Capa: Camadas horizontais, muito bem estratificadas, de siltitos da Formação Corumbataí, em corte da Rodovia SP280, Pres. Castello Branco, porção central da pista capital-interior (Foto CDR Carneiro). 